\title{
The Small Octagons of Maximal Width
}

\author{
Charles Audet · Pierre Hansen • Frédéric Messine • Jordan Ninin
}

Received: 17 May 2012 / Revised: 7 November 2012 / Accepted: 11 February 2013 /

Published online: 19 March 2013

(C) Springer Science+Business Media New York 2013

\begin{abstract}
The paper answers an open problem introduced by Bezdek and Fodor (Arch. Math. 74:75-80, 2000). The width of any unit-diameter octagon is shown to be less than or equal to $\frac{1}{4} \sqrt{10+2 \sqrt{7}}$ and there are infinitely many small octagons having this optimal width. The proof combines geometric and analytical reasoning as well as the use of a recent version of the deterministic and reliable global optimization code IBBA based on interval and affine arithmetics. The code guarantees a certified numerical accuracy of $1 \times 10^{-7}$.
\end{abstract}

Keywords Polygon · Octagon · Width · Diameter

\section{Audet $(\varangle)$}

GERAD and Département de Mathématiques et de Génie Industriel, École Polytechnique de Montréal, C.P. 6079, Succ. Centre-ville, Montreal, QC H3C 3A7, Canada

e-mail: Charles.Audet@gerad.ca

URL: www.gerad.ca/Charles.Audet

P. Hansen

GERAD and Département des Méthodes Quantitatives, HEC Montréal, 3000 Chemin de la Côte Sainte Catherine, Montreal, QC H3T 2A7, Canada

e-mail: Pierre.Hansen@gerad.ca

URL: www.gerad.ca/Pierre.Hansen

\section{F. Messine}

ENSEEIHT-IRIT, University of Toulouse, 2 rue Camichel BP 7122, 31071 Toulouse Cedex 7, France e-mail: Frederic.Messine@n7.fr

URL: http://www.n7.fr/ messine

\section{J. Ninin}

IHSEV Team, LAB-STICC, UMR-CNRS 3162, ENSTA-Bretagne, 2 Rue Franois Verny, 29200 Brest, France

e-mail: Jordan.Ninin@ensta-bretagne.fr

URL: http://sites.google.com/site/jordanninin 


\section{Introduction}

Consider a polygon with $n$ sides and unit diameter, called small polygon by Graham [11]. Which has the maximum width? This question was addressed and partially solved by Bezdek and Fodor in [8]. These authors showed that for all odd values of $n$, the optimal solution is the regular polygon, and for $n \neq 2^{s}$ (with $s \geq 3$ and $s$ integer) solutions are based on Reuleaux polygons [22]. They also solved the quadrilateral case, whose solution is shown to be composed of an equilateral triangle of side length one with an additional vertex in the associated Reuleaux triangle such that the quadrilateral polygon remains convex, leading to infinitely many optimal quadrilaterals. For arbitrary values of $n$, they proved that the value $\cos (1 / 2 n)$ is an upper bound on the polygon's width. In a recent paper [16], Mossinghoff studies enumeration of solutions for $n \neq m 2^{s}$ with $s \geq 0$ and $m$ odd and presents other optimal solutions based on irregular circumscribing Reuleaux polygons.

The first open case is solved in this paper with an numerical certification of $10^{-7}$ and the main result consists of finding a family of small octagon of largest width. The following definitions are useful. The diameter graph of an $n$-sided small polygon is a graph with $n$ nodes (one for each vertex of the polygon), and the edges are composed of the unit-length diagonals. If a node of a graph belongs to a single edge, then that edge is said to be pending. Thus, the main result of this paper is the following:

Theorem 1.1 There is a unit-diameter octagon of maximal width whose diameter graph consists of a cycle of length five with three non-consecutive pending edges. The optimal width is $\hat{w}=\frac{1}{4} \sqrt{10+2 \sqrt{7}} \approx 0.977608773427833$.

The left part of Fig. 1 illustrates an optimal octagon of Theorem 1.1. The illustrated octagon possesses a vertical axis of symmetry. In all figures of this work, dotted lines represent sides of an octagon and full lines represent diameters, i.e., unit-length diagonals. The angles represented by $\theta$ in the figure are identical, and elementary geometry implies that the equation $\sin (\theta)=\cos (\theta)-\frac{1}{2}$ must be satisfied. The unique solution to this trigonometric relation in the interval of interest is $\theta=\arctan \left(\frac{1}{3}(4-\right.$ $\sqrt{7})$ ), and the width of the corresponding octagon is simply $\hat{w}=\cos (\theta / 2)$.

The right part of Fig. 1 zooms in on the three top right vertices of the octagon. The curves represent arcs of circles with radii 1 and $\hat{w}$, centered at the opposite vertex of the octagon. Close inspection of the figure reveals that both sides represented by dotted lines are not tangent to the circle of radius $\hat{w}$. This implies that the central vertex may be slightly moved without altering neither the diameter nor the width of the octagon. Therefore, there are infinitely many small octagons of width equal to $\hat{w}$. The same reasoning applies to the symmetrical pending edge on the top-left part of Fig. 1. However, moving the vertical pending edge will decrease the width of the octagon.

The proof of Theorem 1.1 is given in the next two sections. It involves exploiting bounds on lengths of sides, heights, and diagonals. Section 2 proves a series of lemmas leading to a proposition guaranteeing that the optimal octagon has one of two configurations. The interval branch-and-bound code IBBA is applied in Sect. 3 to numerically solve these configurations. Related solved and unsolved problems are briefly discussed in the concluding section. 

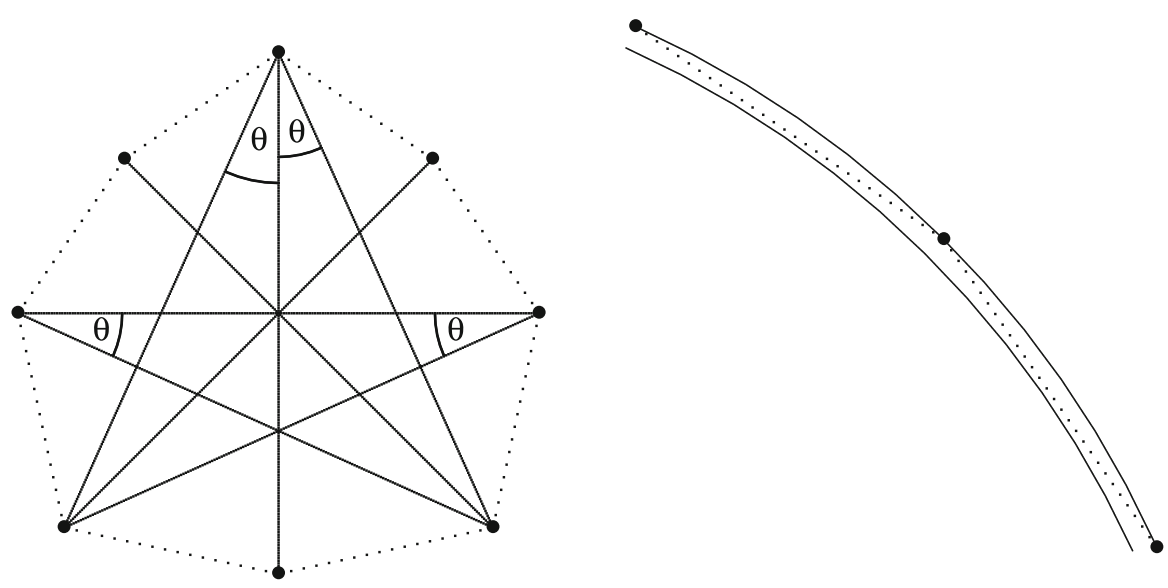

Fig. 1 A small octagon of maximal width (left). Zoom in on the three top right vertices (right)

\section{Bounding Attributes of Small Octagons}

The following notation is used throughout the paper. Let $v_{i}=\left(x_{i}, y_{i}\right) \in \mathbb{R}^{2}$, for $i \in N=\{1,2, \ldots, 8\}$, denote the clockwise cartesian coordinates of the vertices of a small octagon whose width is greater than or equal to $\hat{w}=\frac{1}{4} \sqrt{10+2 \sqrt{7}}$. Sums or differences of the indices of the coordinates are taken modulo 8. For each $i \in N$, the length of the side $\overline{v_{i} v_{i+1}}$ is denoted by $c_{i}$.

Definition 2.1 The height $h_{i}$ associated to the side $\overline{v_{i} v_{i+1}}$ for $i \in N$ is the distance to the furthest vertex - whose index is denoted by $k_{i}$ - of the octagon to the line supporting the side $\overline{v_{i} v_{i+1}}$.

The width of an octagon is the value $W=\min \left\{h_{i}: i \in N\right\}$.

Combining this definition with our assumption that the width of our octagon is at least $\hat{w}$ implies that $h_{i} \geq \hat{w}$ for every $i \in N$.

\subsection{Bounds on the Width}

The question of maximizing the width $W$ under the constraint of unit perimeter $P$ is studied in $[7,10]$ and the question of maximizing the perimeter $P$ under the constraint of unit diameter $D$ is studied in [5]. For the case where $n=8$, these results ensure that

$$
\left(\begin{array}{cl}
\max & W \\
\text { s.t. } & P=1
\end{array}\right) \leq \frac{1}{16} \cot \left(\frac{\pi}{16}\right) \quad \text { and } \quad\left(\begin{array}{cl}
\max & P \\
\text { s.t. } & D=1
\end{array}\right) \leq 3.1211472 .
$$

By combining these two inequalities, it follows that

$$
\hat{w} \leq\left(\begin{array}{cl}
\max & W \\
\text { s.t. } & D=1
\end{array}\right) \leq \frac{3.1211472}{16} \times \cot \left(\frac{\pi}{16}\right)<0.980693
$$


which leads to an improvement by almost $10^{-4}$ of Bezdek and Fodor's [8] upper bound of $\cos (\pi / 16)$ on the optimal width of the octagon.

\subsection{Bounds on the Length of the Sides and Diagonals}

The next lemma bounds the length of the sides of the octagon.

Lemma 2.2 Let $c_{i}$ and $c_{j}$ be the lengths of two distinct sides. If $c_{i} \leq c_{j}$, then

$$
c_{\min } \leq c_{i} \leq c_{\max } \text { and } c_{\min }+c_{\max } \leq 2 c_{j},
$$

where $c_{\max }=\frac{1}{2} \sqrt{6-2 \sqrt{7}}$ and $c_{\min }=16 \hat{w} \times \tan \left(\frac{\pi}{16}\right)-7 c_{\max }$.

Proof For the side $\overline{v_{i} v_{i+1}}$ of length $c_{i}$, the corresponding height $h_{i}$ is bounded above by $\sqrt{1-\left(\frac{c_{i}}{2}\right)^{2}}$, otherwise either $v_{i}$ or $v_{i+1}$ would be at distance exceeding the diameter $D=1$ from $p_{i}$. Therefore,

$$
c_{i}^{2} \leq 4\left(1-\hat{w}^{2}\right)=4\left(1-\left(\frac{1}{16}(10+2 \sqrt{7})\right)\right)=\frac{1}{4}(6-2 \sqrt{7})=c_{\max }^{2}
$$

Combining the fact that the perimeter $P$ satisfies $P \leq c_{i}+7 c_{\max }$ with Eq. (1) yields

$$
\hat{w} \leq\left(\begin{array}{cl}
\max & W \\
\text { s.t. } & P \leq c_{i}+7 c_{\max }
\end{array}\right) \leq \frac{c_{i}+7 c_{\max }}{16} \cot \left(\frac{\pi}{16}\right)
$$

which completes the first part of the proof.

A similar argument on $P \leq c_{i}+c_{j}+6 c_{\max }$ gives $c_{\min }+c_{\max } \leq c_{i}+c_{j} \leq 2 c_{j}$, which concludes the proof.

The following lemma shows that the lengths of diagonals of two consecutive vertices cannot be both greater than or equal to $\hat{w}$.

Lemma 2.3 For $i \neq j \in N$, the length of $\overline{v_{i} v_{j+1}}$ and $\overline{v_{i+1} v_{j}}$ cannot be both greater than or equal to $\hat{w}$.

Proof Suppose that the diagonal lengths $\ell_{1}=\left|\overline{v_{i} v_{j+1}}\right|$ and $\ell_{2}=\left|\overline{v_{i+1} v_{j}}\right|$ satisfy $\hat{w} \leq \ell_{1} \leq 1, \hat{w} \leq \ell_{2} \leq 1$ and that $c_{i} \leq c_{j}$. By rotating the octagon if necessary, let $v_{i}=\left(\frac{c_{i}}{2}, 0\right)$ and $v_{i+1}=\left(-\frac{c_{i}}{2}, 0\right)$ be the cartesian coordinates of the vertices $v_{i}$ and $v_{i+1}$. Now, as $\hat{w} \leq \ell_{1} \leq 1$, it follows that $\hat{w}^{2} \leq\left(x_{j+1}-\frac{c_{i}}{2}\right)^{2}+y_{j+1}^{2} \leq 1$. Also, $\mid \overline{v_{i+1} v_{j+1} \mid}=\left(x_{j+1}+\frac{c_{i}}{2}\right)^{2}+y_{j+1}^{2} \leq 1$ as the octagon's diameter is one. The arcs in Fig. 2 represent circles of radius $\hat{w}$ and 1 centered at $v_{i}$ and $v_{i+1}$. The shaded area of the figure represents the region in which the vertex $v_{j+1}$ lies.

Similar bounds on the cartesian coordinates of $v_{j}$ can easily be derived. But as $v_{j}$ and $v_{j+1}$ are consecutive vertices, it follows that the largest value that $x_{j+1}$ may take 
Fig. 2 An impossible

configuration

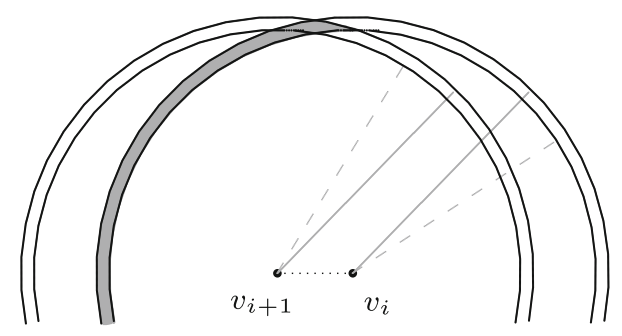

is when $v_{j+1}$ is at unit distance from $v_{i+1}$ and at distance $\hat{w}$ from $v_{i}$. This corresponds to the rightmost point of the shaded area in the figure. This situation arises when

$$
\hat{w}^{2}=\left(x_{j+1}-\frac{c_{i}}{2}\right)^{2}+y_{j+1}^{2} \text { and }\left(x_{j+1}+\frac{c_{i}}{2}\right)^{2}+y_{j+1}^{2}=1,
$$

i.e., when $x_{j}=\frac{1-\hat{w}^{2}}{2 c_{i}}$. Similarly, the least value that $x_{j}$ may take is $-\frac{1-\hat{w}^{2}}{2 c_{i}}$ and it follows that the length $c_{j}$ or the side $\overline{v_{j} v_{j+1}}$ satisfies $c_{j} \leq \frac{1-\hat{w}^{2}}{c_{i}}$.

By numerically evaluating the values $c_{\min }, c_{\max }$, and $\hat{w}$, Lemma 2.2 implies

$$
0.096<c_{\min }\left(c_{\min }+c_{\max }\right) \leq 2 c_{i} c_{j} \leq 2\left(1-\hat{w}^{2}\right)<0.089
$$

This contradiction implies that at most one of the two diagonals may exceed $\hat{w}$ in length.

\subsection{The Height Associated to a Side}

The next result shows that the index $k_{i}$ of the furthest vertex associated to side $\overline{v_{i} v_{i+1}}$ cannot be the first or second neighbor of the side.

Lemma 2.4 For any $i \in N, k_{i} \in\{i+4, i+5\}$.

Proof On the one hand, Lemma 2.2 ensures that the length of the each side of the octagon is less than $c_{\min }<0.43$. On the other hand, the distance from the $i$ th side to $v_{k_{i}}$ is not less than $\hat{w}>0.97>2 c_{\min }$. It follows that $k_{i}$ cannot be $i+2, i+3, i+6$, or $i+7$.

The following lemma provides additional properties that the furthest vertices must satisfy. 
Lemma 2.5 For any $i \in N, k_{i}=i+4$ if and only if $k_{i+4}=i+1$, and $k_{i}=i+5$ if and only if $k_{i+4}=i$.

Proof If the two sides $\overline{v_{i} v_{i+1}}$ and $\overline{v_{i+4} v_{i+5}}$ were parallel, then both distances $\left|\overline{v_{i} v_{i+5}}\right|$ and $\left|\overline{v_{i+1} v_{i+4}}\right|$ would be at least $\hat{w}$, which would contradict Lemma 2.3. Therefore, the two sides $\overline{v_{i} v_{i+1}}$ and $\overline{v_{i+4} v_{i+5}}$ are not parallel.

Let $P$ be the intersection point of the two support lines of the sides $\overline{v_{i} v_{i+1}}$ and $\overline{v_{i+4} v_{i+5}}$. If $v_{i+1}$ belongs to the segment $\overline{v_{i} P}$, then necessarily $v_{i+4}$ belongs to the segment $\overline{P v_{i+5}}$ because the vertices are labeled in a clockwise way. It follows that the furthest vertex from the support line containing the side $\overline{v_{i} v_{i+1}}$ is $v_{i+5}$, and the furthest vertex from the support line containing the side $\overline{v_{i+4} v_{i+5}}$ is $v_{i}$, i.e., $k_{i}=i+5$ and $k_{i+4}=i$.

A similar development leads to $k_{i}=i+4$ and $k_{i+4}=i+1$ when $v_{i}$ belongs to the segment $\overline{v_{i+1} P}$.

Let us define the height graph of the octagon as follows. The vertices of the graph are the vertices $N=\{1,2, \ldots, 8\}$ of the octagon, and the undirected edges are $\left\{\left(i, k_{i}\right): i \in\right.$ $N\} \cup\left\{\left(i+1, k_{i}\right): i \in N\right\}$. By construction, the length of each diagonal corresponding to the edges of the height graph is greater than or equal to $\hat{w}$.

Proposition 2.6 The height graph is either composed of a cycle of length five with three pending edges originating from three non-consecutive vertices (Fig. 3e), or a cycle of length seven with one pending edge (Fig. 3d).

Proof Without any loss of generality, Lemma 2.5 allows us to assume that $k_{1}=5$ and $k_{5}=2$. This means that the furthest vertex from the side $\overline{v_{1} v_{2}}$ is $v_{5}$ and the furthest from $\overline{v_{5} v_{6}}$ is $v_{2}$ Therefore, the edges $\left(v_{1}, v_{5}\right),\left(v_{5}, v_{2}\right)$, and $\left(v_{2}, v_{6}\right)$ belong to the height graph as represented in Fig. 3a. In all the figures, the dashed lines represent diagonals of length greater than or equal to $\hat{w}$.

Figure 3 a possesses an axis of symmetry going through the sides $\overline{v_{3} v_{4}}$ and $\overline{v_{7} v_{8}}$ and so, without any loss of generality we can assume that $k_{3}=7$ and $k_{7}=4$, as represented by Fig. $3 b$.

Figure $3 \mathrm{~b}$ has an axis of symmetry going through the sides $\overline{v_{4} v_{5}}$ and $\overline{v_{8} v_{1}}$ and so, without any loss of generality we can assume that $k_{4}=1$ and $k_{8}=4$, as represented by Fig. 3b.

Figure $3 \mathrm{c}$ is not symmetrical with respect to the sides $\overline{v_{2} v_{3}}$ and $\overline{v_{6} v_{7}}$. Therefore, there are two possibilities: either $k_{2}=6$ or $k_{6}=3$, as represented by Fig. 3d, or $k_{2}=7$ and $k_{6}=2$, as represented by Fig. $3 \mathrm{e}$.

Figure $3 \mathrm{~d}$ consists of a cycle of length seven with one pending edge, and Fig. $3 \mathrm{e}$ is a cycle of length five with three pending edges originating from non-consecutive vertices.

\subsection{Unit Pending Edges}

Proposition 2.6 showed that an optimal octagon has at least one pending edge in its height graph. The next result shows that the length of these edges may be set to one. 

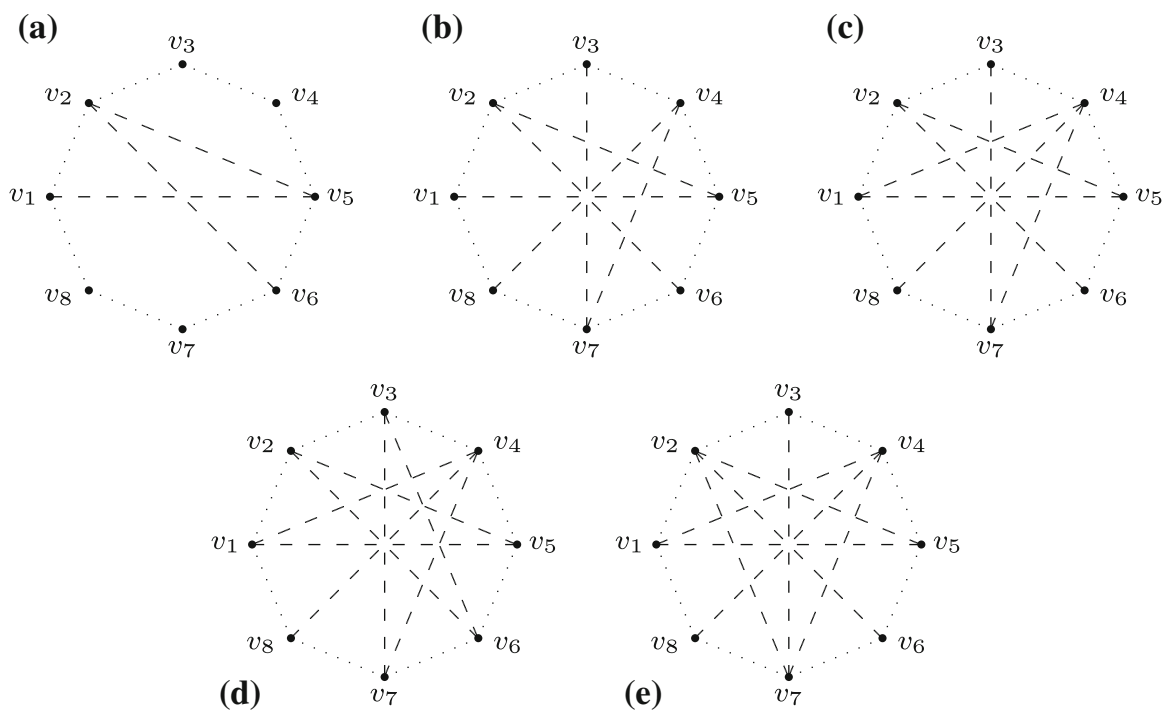

Fig. 3 Partial height graphs for the proof of Proposition 2.6

Proposition 2.7 There is a small octagon whose width is greater than or equal to $\hat{w}$ and where the pending edges are diameters.

Proof Let $\overline{v_{i} v_{j}}$ for $j \in\{i+3, i+4, i+5\}$ be edges of the height graph, and by rotating if necessary, suppose that $v_{i}=(0,0)$ and $v_{i+4}=\left(x_{i+4}, 0\right)$, where $x_{i} \geq \hat{w}$ is the length of the pending edge.

Consider the situation where $x_{i+4}<1$. We will analyze the effect of modifying the octagon by varying the value of $x_{i+4}$ while conserving the position of all other vertices. Let us increase the value of $x_{i+4}$ as much as possible, while retaining the condition that the diameter of the octagon is equal to one.

This means that there exists an index $j$ such that $\left|\overline{v_{j} v_{i+4}}\right|=1$. Recall that the original octagon has optimal width. Therefore, there are two possibilities:

- The width of the octagon remains the same or increases.

Lemma 2.3 ensures that $j=i$, and consequently the width of the transformed octagon is at least as large as the original one.

- The width of the octagon decreases.

In this situation, let us decrease the value of $x_{i+4}$ until $v_{i+4}$ belongs to the line segment joining $v_{i+3}$ and $v_{i+5}$. The width of the octagon would then increase, and the distances from $v_{i+4}$ to all other vertices would still be bounded above by 1. This transformation leads to a unit-diameter heptagon of width greater than $\hat{w}$. This contradicts the result of Bezdek and Fodor [8] which states that the optimal heptagon width is $\cos (\pi / 14)<\hat{w}$.

Therefore, the pending vertices may be moved in such a way that the pending edge's length is one, without decreasing the width of the octagon. 


\section{Numerical Results}

The previous geometric and analytical reasoning gives a characterization of the globally optimal octagons. The two configurations illustrated in Fig. 3d, e remain to be analyzed.

This section uses an interval branch-and-bound algorithm to eliminate the remaining cases by solving nonlinear and non-convex optimization problems. The main advantages of such an algorithm is that all the numerical rounding errors are taken into account during the computation, and therefore the resulting solutions satisfy a certified and reliable accuracy. If there is no solution, a certificate of infeasibility is guaranteed.

\subsection{An Interval Branch-and-Bound Algorithm}

The global optimization algorithm used here is a Fortran 90/95 implementation of a branch-and-bound method, named IBBA (interval branch-and-bound algorithm), where bounds are computed using interval and affine arithmetics.

Interval analysis was introduced by Moore [15] to control the propagation of numerical errors due to floating point arithmetic. Thus, Moore proposes to enclose any real value by an interval whose the bounds are the two closest floating point numbers. Then expanding the classical operations-addition, subtraction, multiplication, and division-into intervals defines interval arithmetic [23]. A straightforward generalization allows computation of reliable bounds (excluding the problem of numerical errors) of a function over a hypercube (or box) defined by an interval vector. Moreover, classical tools of analysis such as Taylor expansions can be used together with interval arithmetic to compute more precise bounds [15]. Other bounding techniques, such as affine arithmetic computations [9], use linear dependency to improve the bounds.

The principle of IBBA is based on a branch-and-bound technique. At each iteration, the domain under study is bisected to improve the computation of bounds. Boxes are eliminated if and only if it is certified that no point in the box can produce a better solution than the current best one, or that at least one constraint cannot be satisfied by any point in such a box.

To accelerate convergence, constraint propagation techniques are used at each iteration of IBBA, see $[13,18]$ for details. The principle is to use the implicit relations between the variables (induced by the constraints) to prune the width of a box.

Another accelerating technique, named Affine Relaxation Technique, is added [18, 20]. This procedure computes reliable bounds and certificates of infeasibility for each sub-box. This linear relaxation are generated using an extension of affine arithmetic $[12,14]$. To ensure the numerical reliability of the solutions provided by a linear solver (here, we used CPLEX), a method from [17] is used.

These two techniques improve the performance of IBBA allowing us to solve the next problems using the new version of IBBA described in [18-20]. 


\subsection{A Nonlinear Formulation of the Optimal Octagon}

A general formulation of our problem of finding the small octagon of maximal width is given by

$$
\begin{array}{ll}
\max _{\substack{v_{i}=\left(x_{i}, y_{i}\right) \\
i \in N}} & W=\min _{i} \max _{j \neq i, i+1} \frac{\left|\left(y_{i+1}-y_{i}\right) x_{i}+\left(x_{j}-x_{j+1}\right) y_{j}+x_{j+1} y_{j}-x_{j} y_{j+1}\right|}{\left|\overline{v_{i} v_{i+1}}\right|}, \\
\text { s.t. } & D=\max _{1 \leq i<j \leq 8}\left|\overline{v_{i} v_{j}}\right|=1 .
\end{array}
$$

Unfortunately, this general formulation of the problem cannot be solved directly in a reasonable amount of time. However, the results derived in the previous section may be exploited to add constraints to the problem and to reduce the feasible domain.

The simple additional constraints $\hat{w} \leq W \leq 0.980693$ may be added as they represent valid lower and upper bounds on the width of the octagon, as shown in Sect. 2.1. Other constraints are derived from Lemma 2.2 which give lower and upper bounds on the side length. Proposition 2.6 imposes conditions over some diameters and simplify the computation of the heights associated to all of the sides. Let $h_{i}\left(v_{j}\right)$ denote the height distance between the support line of $\overline{v_{i} v_{i+1}}$ and the vertex $v_{j}$ :

$$
h_{i}\left(v_{j}\right)=\frac{\left|\left(y_{i+1}-y_{i}\right) x_{j}+\left(x_{i}-x_{i+1}\right) y_{j}+x_{i+1} y_{i}-x_{i} y_{i+1}\right|}{\sqrt{\left(x_{i+1}-x_{i}\right)^{2}+\left(y_{i+1}+y_{i}\right)^{2}}}
$$

Additional notation needs to be introduced to model the graphs represented in Fig. 3d, e. Let $I_{1}$ denote the set of indices corresponding to the dashed diagonals, $I_{2}$ the indices of the pending edges, and $I_{3}$ the indices describing the height relative to a side of the octagon. Using the notation from Sect. 2.3, this last set is defined as $I_{3}=\left\{\left(i, k_{i}\right), \forall i \in N\right\}$.

For Fig. 3d, the set of indices are

$$
\left\{\begin{array}{l}
I_{1}=\{(1,4),(1,5),(2,5),(2,6),(3,6),(3,7),(4,7)\} \\
I_{2}=\{(4,8)\} \\
I_{3}=\{(1,5),(2,6),(3,7),(4,1),(5,2),(6,3),(7,4),(8,4)\}
\end{array}\right.
$$

and for Fig. 3e they are

$$
\left\{\begin{array}{l}
I_{1}=\{(1,4),(1,5),(2,5),(2,7),(4,7)\} \\
I_{2}=\{(2,6),(3,7),(4,8)\} \\
I_{3}=\{(1,5),(2,7),(3,7),(4,1),(5,2),(6,2),(7,4),(8,4)\}
\end{array}\right.
$$

Proposition 2.7 ensures that the pending edges may be considered as diameters, and so, we can set the coordinates of two vertices: $v_{8}=(0,0)$ and $v_{4}=(0,1)$. The variable $w$ and constraints are introduced to model the minmax function from the objective and to compute the height of the octagon. This leads to the following model 
with appropriate sets $I_{1}, I_{2}$, and $I_{3}$ :

$$
\begin{array}{cll}
\max _{\substack{w \in \mathbb{R}, v_{i}=\left(x_{i}, y_{i}\right), i \in N \backslash\{4,8\} \\
\text { s.t. }}} & w & \\
& c_{\min } \leq\left|\overline{v_{i} v_{i+1}}\right| \leq c_{\max } & \forall i \in N, \\
& \hat{w} \leq\left|\overline{v_{i} v_{j}}\right| \leq 1 & \forall(i, j) \in I_{1}, \\
& \left|\overline{v_{i} v_{j}}\right|=1 & \forall(i, j) \in I_{2}, \\
& h_{i}\left(v_{j+1}\right) \leq h_{i}\left(v_{j}\right) \geq h_{i}\left(v_{j-1}\right) & \forall(i, j) \in I_{3}, \\
& w \leq h_{i}\left(v_{j}\right) \leq 1, & \forall(i, j) \in I_{3}, \\
& \hat{w} \leq w \leq 0.980693, & \\
& y_{3} \geq y_{2} \geq y_{1} \leq y_{7} \leq y_{6} \leq y_{5} . &
\end{array}
$$

The model is then further simplified by squaring the inequalities involving absolute values or square roots. Denominators are multiplied on each side of the inequalities. This leads to a nonlinear model with 13 continuous variables and 65 non-convex constraints. The initial domain is $\left(v_{1}, v_{2}, v_{3}\right) \in([0,1] \times[0,1])^{3}$ and $\left(v_{5}, v_{6}, v_{7}\right) \in$ $([-1,0] \times[0,1])^{3}$.

Computations are performed using IBBA on an AMD64 AthlonII 2.4 GHz computer with 2 GB of RAM.

For the case (3d), IBBA delivers a certificate of infeasibility in $1.40 \mathrm{~s}$ and 742 iterations. This confirms that there is no small octagon under configuration of Fig. $3 \mathrm{~d}$ having a width greater than $\frac{1}{4} \sqrt{10+2 \sqrt{7}}$.

The case (3e) requires more computation as there are infinitely many global solutions due to the pending edges. An accuracy of $10^{-4}$ is guaranteed in 4.75 min and an accuracy of $10^{-5}$ requires $11.13 \mathrm{~min}$.

Finally, with one day of computation, we certify that the optimal width lies in the interval [0.97760877, 0.97760980], which guarantees an accuracy of $1.03 \times 10^{-6}$. By replacing the lower bound on the width $w \geq \hat{w}$ by $w \geq\left(\hat{w}+1 \times 10^{-7}\right)$ in Problem (4), IBBA certifies in $7 \mathrm{~h}$ and $33 \mathrm{~min}$ that there does not exist an better solution.

In summary, the global maximum value is guaranteed to be $\hat{w}$ with a certified numerical accuracy of $1 \times 10^{-7}$.

\section{Conclusion and Discussion}

The width of any small octagon is shown to be less than or equal to the value $\hat{w}=$ $\frac{1}{4} \sqrt{10+2 \sqrt{7}}$, with a guaranteed numerical accuracy of $1 \times 10^{-7}$. The numerical certification was ensured by a new version of the IBBA code $[18,20]$. For $n$-sided polygons, the next open case is the hexadecagon $\left(n=2^{4}=16\right.$ sides) [8] .

There are infinitely many small octagons whose width is exactly $\hat{w}$. Some, but not all of them, have a vertical axis of symmetry. Among all these solutions, the one having the maximal area is unique, and it is the symmetrical one depicted in Fig. 1 where four of its diameters cross at an interior point of the octagon. Elementary computations reveal that the value of the area is $\sqrt{2} / 2$, which is the exact same value as that of the regular small octagon. 
Three related questions involving small octagons were answered in previous study: (i) maximization of the area [2], (ii) maximization of the length of the perimeter [5], (iii) maximization of the length of the perimeter for octagon with equal sides [3]. These questions were also solved by combining geometric reasoning with the use of global optimization codes. The optimality proofs of these four optimal octagons differ, leading to the study of different ways to enumerate, formulate, and solve subproblems.

In two recent survey articles [4,6], optimal problems and their corresponding complete or partial solutions involving area, perimeter, diameter, width, and sum of distances of (convex) polygons were presented. Problems involving the width include the following ones. Mossinghoff [16] recalls an old result of Pál from [21] (concerning the solution of the Kakeya problem in the convex case, see also p. 221 of [24]) which answers the question of maximizing the width of a convex figure with a fixed area: the optimal solution is the equilateral triangle. Another problem concerns the maximization of the width of unit-perimeter polygons. The question was partially answered by Gabashkov in [10] and complementary results were presented in [7]. The remaining open cases are those where the number of edges $n$ is a power of 2 . The quadrilateral case is solved in [7] and the first open problem occurs when $n=8$. Finally, the three questions of maximizing the perimeter, diameter, and area of equilateral unit-width convex polygons are studied in [1]. When the number of sides is even, the optimal value is trivially unbounded. When the number of sides is odd, the same polygon is shown to be optimal for the three problems.

Acknowledgment The work of the first author was supported by NSERC Grant 239436-2010.

\section{References}

1. Audet, C., Ninin, J.: Maximal perimeter, diameter and area of equilateral unit-width convex polygons. J. Glob. Optim. (2011). doi:10.1007/s10898-011-9780-4

2. Audet, C., Hansen, P., Messine, F., Xiong, J.: The largest small octagon. J. Combin. Theory Ser. A 98(1), 46-59 (2002)

3. Audet, C., Hansen, P., Messine, F., Perron, S.: The minimum diameter octagon with unit-length sides: Vincze's wife's octagon is suboptimal. J. Combin. Theory Ser. A 108(1), 63-75 (2004)

4. Audet, C., Hansen, P., Messine, F.: Extremal problems for convex polygons. J. Glob. Optim. 38(2), 163-179 (2007)

5. Audet, C., Hansen, P., Messine, F.: The small octagon with longest perimeter. J. Comb. Theory Appl. Ser. A 114(1), 135-150 (2007)

6. Audet, C., Hansen, P., Messine, F.: Extremal problems for convex polygons-an update. In: Pardalos, P.M., Coleman, T.F. (eds.) Lectures on Global Optimization, Fields Institute Communications, vol. 55, pp. 1-16. American Mathematical Society, Providence, RI (2009).

7. Audet, C., Hansen, P., Messine, F.: Isoperimetric polygons of maximum width. Discrete Comput. Geom. 41(1), 45-60 (2009)

8. Bezdek, A., Fodor, F.: On convex polygons of maximal width. Arch. Math. 74, 75-80 (2000)

9. de Figueiredo, L., Stolfi, J.: Affine arithmetic: concepts and applications. Numer. Algorithms 37(1-4), 147-158 (2004)

10. Gashkov, S.: Inequalities for the area and perimeter of a convex polygon. Kwant 10, 15-19 (1985). In Russian: FIGA1 FIGA2 10, 15-19 (1985).

11. Graham, R.L.: The largest small hexagon. J. Combin. Theory 18, 165-170 (1975)

12. Messine, F.: Extensions of affine arithmetic: application to unconstrained global optimization. J. Univ. Comput. Sci. 8(11), 992-1015 (2002) 
13. Messine, F.: Deterministic global optimization using interval constraint propagation techniques. RAIRO Oper. Res. 38(4), 277-293 (2004)

14. Messine, F., Touhami, A.: A general reliable quadratic form: an extension of affine arithmetic. Reliab. Comput. 12(3), 171-192 (2006)

15. Moore, R.E.: Interval Analysis. Prentice-Hall Inc., Englewood Cliffs, NJ (1966)

16. Mossinghoff, M.J.: Enumerating isodiametric and isoperimetric polygons. J. Comb. Theory Ser. A 118(6), 1801-1815 (2011)

17. Neumaier, A., Shcherbina, O.: Safe bounds in linear and mixed-integer linear programming. Math. Program. Ser. A 99(2), 283-296 (2004)

18. Ninin, J.: Optimisation globale basée sur l'analyse d'intervalles: relaxation affine et limitation de la mémoire. PhD thesis, Institut National Polytechnique de Toulouse (2010). http://ethesis.inp-toulouse. fr/archive/00001477/01/Ninin.pdf

19. Ninin, J., Messine, F.: A metaheuristic methodology based on the limitation of the memory of interval branch and bound algorithms. J. Glob. Optim. 50, 629-644 (2010)

20. Ninin, J., Messine, F., Hansen, P.: A reliable affine relaxation method for global optimization. Technical Report G-2010-31, GERAD (2010). http://www.optimization-online.org/DB_HTML/2012/10/3650. html

21. Pál, J.: Ein Minimumproblem für Ovale. Math. Ann. 83, 311-319 (1921)

22. Reuleaux, F.: The Kinematics of Machinery. Dover, New York, NY (1963)

23. Revol, N.: Standardized interval arithmetic and interval arithmetic used in libraries. In: Fukuda, K., van der Hoeven, J., Joswig, M., Takayama, N. (eds.) Mathematical Software-ICMS 2010, vol. 6327, pp. 337-341. Springer, Berlin (2010) WOS:000286346100054

24. Yaglom, I.M., Boltyanskiǔ, V.G.: Convex Figures (Translated by P.J. Kelly, L.F. Walton, Lib. Math. Circle, vol. 4) Rinehart and Winston, New York, NY (1961) 